Revista Latinoamericana de la Papa. 24 (2): 54 - 61, 2020

Recibido: 01/10/2020 | Aceptado: 21/11/2020

Publicado en línea: Diciembre 2020

DOI: $10.37066 /$ ralap.v24i2.408

ISSN: 1853-4961

\title{
Promoviendo el comercio de cultivos nativos para mejorar los medios de vida rurales en los Andes
}

\section{André Devaux $^{1 / *}$; Miguel Ordinola ${ }^{2}$; Jorge Andrade-Piedra ${ }^{3}$; Graham \\ Thiele $^{4}$}

\begin{abstract}
Resumen
La producción de papas nativas es una estrategia de sustento importante para la mayoría de las familias rurales andinas. Un enfoque inclusivo de desarrollo de cadenas de valor desarrollado por el Centro Internacional de la Papa (CIP) desencadenó procesos de innovación para mejorar y promover el comercio de papas nativas, mejorando los medios de vida de los pequeños productores y beneficiando a todos los actores de la cadena de valor de papas nativas. Usar las nuevas oportunidades del mercado para el desarrollo requiere una serie de otras innovaciones, incluidas las institucionales y tecnológicas. El enfoque desarrollado inicialmente en Perú también se implementó con éxito en Ecuador y Bolivia y podría expandirse a otros cultivos en otras regiones.
\end{abstract}

Palabras clave adicionales: papas nativas, desarrollo de cadenas de valor inclusivas, investigación agrícola, sistema de innovación.

* Autor para correspondencia. E-mail: andrej.devaux@gmail.com

André Devaux. iD Científico Emérito, Centro Internacional de la Papa (CIP), Louvain-la-Neuve, Bélgica.

2 Miguel Ordinola. iD Asesor Centro Internacional de la Papa (CIP). Docente de Postgrado de la Pontificia Universidad Católica del Perú (PUCP).

3 Jorge Andrade-Piedra. (iD Científico, Centro Internacional de la Papa (CIP), Lima, Perú.

4 Graham Thiele. (D) Director, Programa de Investigación del CGIAR "Roots, Tubers and Bananas", coordinado por CIP, Perú. 


\section{Marketing native crops to improve rural Andean livelihoods Summary}

Native potato production is an important livelihood strategy for most rural Andean families. An inclusive value chain development approach developed by the International Potato Center (CIP) triggered innovation processes to enhance the marketing of native potatoes benefiting small-scale producers and value chain actors and improving their livelihoods. Making use of new market opportunities for development requires an array of other innovations including institutional and technological ones. The approach initially developed in Peru was also successfully implemented in Ecuador and Bolivia and could be expanded to other crops in other regions.

Additional keywords: Native potatoes, inclusive value chain development, agricultural research, Innovation system

\section{Contexto}

Las papas nativas son importantes en las estrategias de subsistencia de la mayoría de las familias andinas pobres. Un enfoque de sistemas orientado a promover el desarrollo pro pobre, a través de cadenas de valor, ha ayudado a identificar oportunidades comerciales, facilitar la

colaboración entre los actores de la cadena de valor y mejorar los flujos de información para aumentar la competitividad.

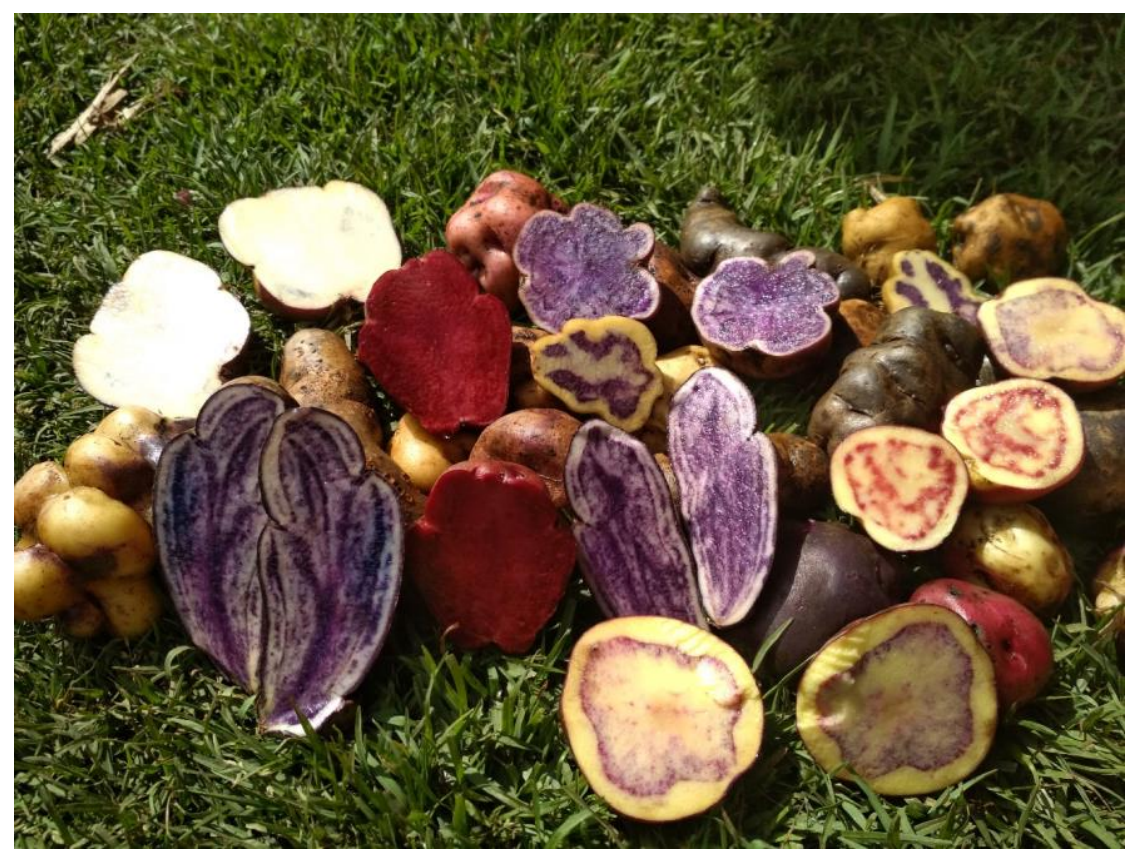

Figura 1. Papas nativas de color @Stef de Haan. 


\section{Intervención del CIP con un enfoque novedoso/innovador}

Las papas han sido un alimento básico de las familias andinas durante miles de años. Debido a que las papas nativas (variedades locales, un recurso fitogenético único) se desarrollan mejor en las alturas (por encima de los 3300 msnm), donde predominan los pequeños agricultores. El Centro Internacional de la Papa (CIP), con sede en Perú, decidió concentrar sus actividades en torno a esas papas para promover innovaciones para el desarrollo inclusivo de la cadena de valor. Los agricultores andinos cultivan más de 3000 variedades de papa nativa, principalmente para satisfacer sus necesidades alimenticias y no para la venta. A menudo, se encuentran en desventaja cuando se trata de hacer negocios en cadenas alimentarias de alta calidad y garantizar el cumplimiento de los estándares, dado el escaso acceso a servicios financieros y de otro tipo y su capacidad limitada de comercialización colectiva. El CIP coordinó con sus socios tanto públicos como privados para desarrollar nuevas cadenas de valor para las papas nativas aplicando el Enfoque Participativo de Cadenas Productivas (EPCP).

El EPCP, es un enfoque de sistemas de innovación que aplica principios de investigación-acción y acción colectiva para fomentar el desarrollo inclusivo de cadenas de valor. Involucra a equipos de investigación multidisciplinarios y a los pequeños agricultores con otros actores de la cadena de valor, con funcionarios públicos y proveedores de servicios, como las ONG, en procesos colectivos facilitados, en los cuales se identifican oportunidades comerciales y se desarrollan innovaciones orientadas al mercado. El proceso de EPCP se implementa durante 12 a 18 meses y consta de tres fases, cada una de las cuales finaliza con un evento público para compartir y promover resultados con los actores de la cadena de valor, proveedores de servicios y formuladores de políticas (Figura 2) (Devaux et al. 2016; Bernet et . 2006).

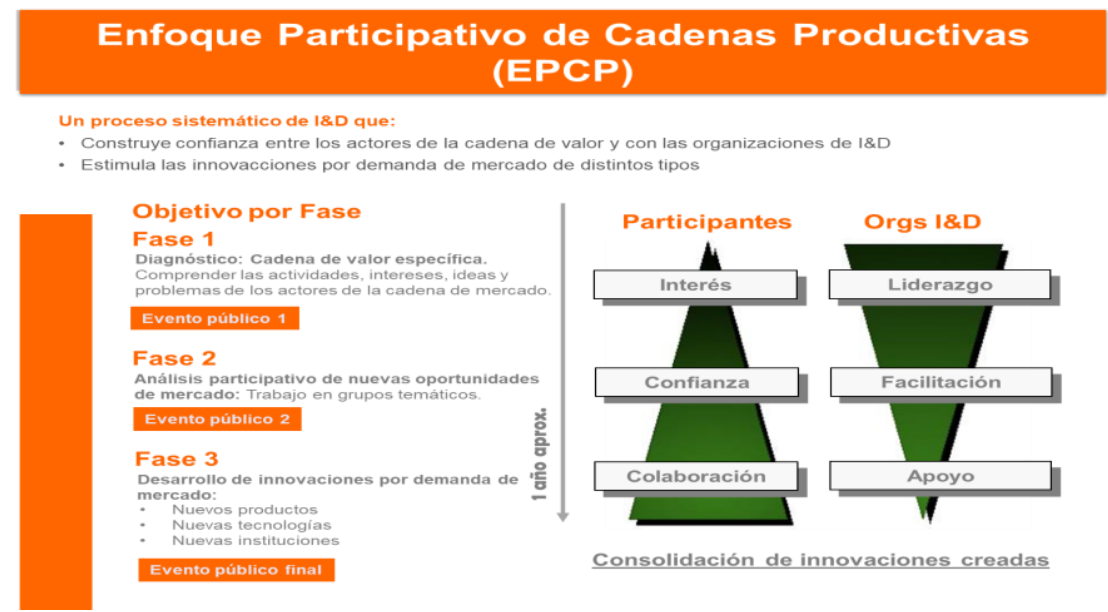

Figura 2. El Enfoque Participativo de Cadenas Productivas (EPCP) 
Para empoderar a los pequeños agricultores, el EPCP se complementa con plataformas locales de concertación que facilitan las interacciones entre los productores de papa, las autoridades locales, el sector privado y los proveedores de servicios. Las plataformas resultan útiles para consolidar procesos de innovación después de un proceso de implementación del EPCP. A través de su énfasis en aprovechar los activos de los pequeños productores y movilizar el conocimiento local y científico, el EPCP es consistente con los enfoques que tengan en cuenta explícitamente las condiciones específicas de mujeres y hombres. El CIP publicó una guía para la integración de género en el EPCP (Mayanja et al. 2016).

El EPCP identificó oportunidades y desarrolló de innovaciones comerciales para nichos de alto valor. Contribuyó a aumentar la visibilidad y el uso de las papas nativas. La selección, el desarrollo de marcas y la creación de nuevos productos procesados de papa nativa, como chips de color natural gourmet, agregó valor y amplió el mercado de estas coloridas y extraordinarias papas (Figura 3). En Perú, el proceso de EPCP demostró ser un "disparador" de innovaciones tecnológicas, institucionales y comerciales.

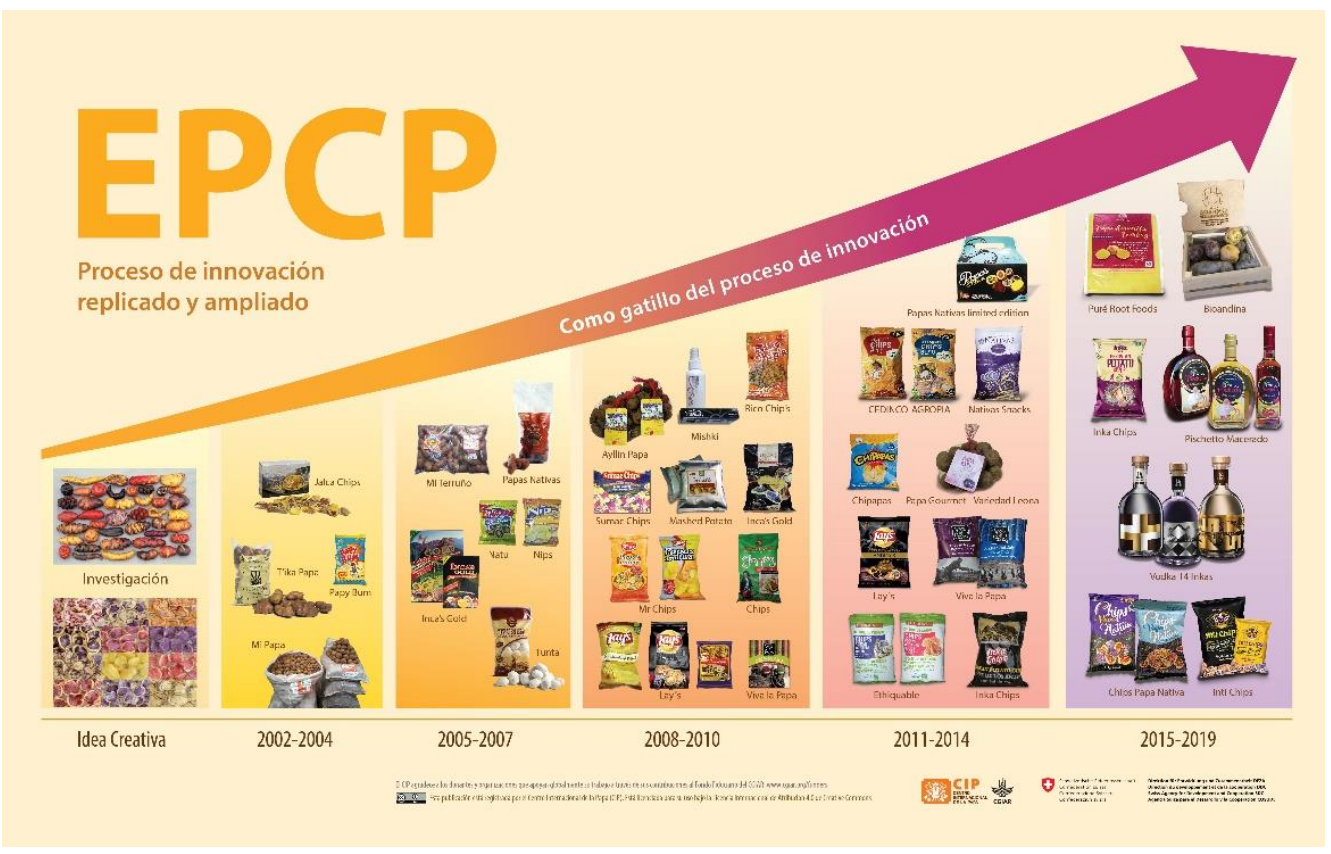

Figura 3. Desarrollo de nuevos productos impulsados por el EPCP en Perú

Estas innovaciones tuvieron varios resultados: redes de actores que estimularon la innovación de la cadena de valor; una fuerte expansión de la demanda de papas nativas por parte de los consumidores urbanos (el volumen de papas nativas vendidas por los agricultores aumentó en más del 70 por ciento a medida que subieron los precios); y cambios de política que reconocen estas papas en el sistema formal de producción de semillas (Devaux et al. 2020). 
La innovación institucional, como el establecimiento de un Día Nacional de la Papa en el Perú, promovió aún más la papa nativa y estimuló su demanda (Ordinola et al. 2011). Las ONG fortalecieron la capacidad de las organizaciones de pequeños productores y mejoraron los vínculos con los agentes del mercado y los procesadores. También, brindaron apoyo técnico para mejorar la calidad y consistencia de la producción y lograr economías de escala. Las mujeres involucradas en la intervención fueron empoderadas para escoger entre varias opciones y tomar decisiones sobre cuestiones de producción y comercialización, lo que les otorgó mayor autoridad para negociar con otros actores de la cadena de valor (Sarapura et al. 2016).

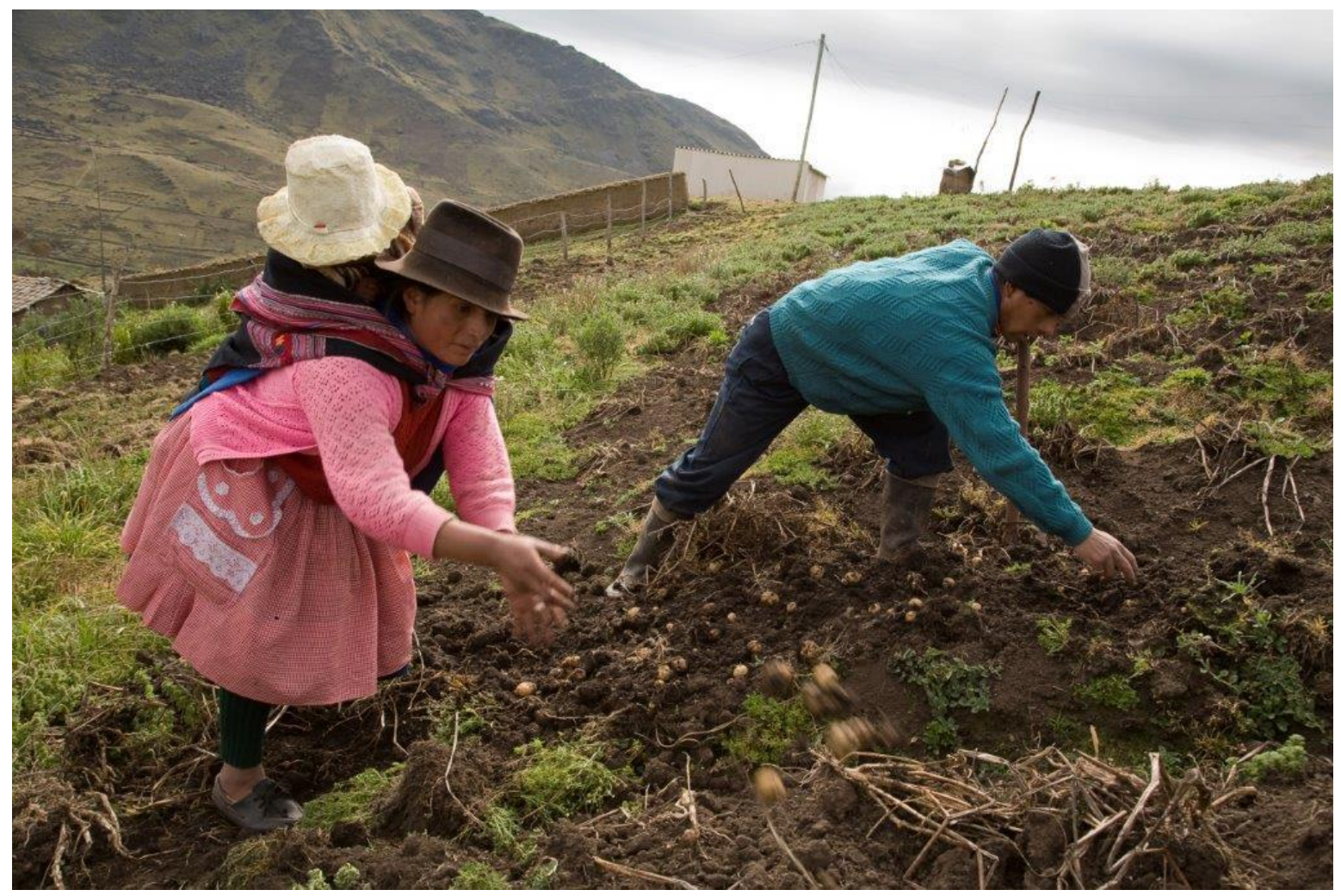

Figura 4. Cosechando papa en los Andes @ J-L Gonterre

El enfoque también se implementó exitosamente en Ecuador y Bolivia. Las experiencias en la difusión del EPCP destacan la importancia de la promoción y el apoyo institucional, el desarrollo de capacidades, el cambio organizacional y el financiamiento. El CIP se apoya en esta experiencia y busca incluir un conjunto más amplio de cultivos dentro de la Iniciativa Andina, para abordar las amenazas de pérdida de agrobiodiversidad, de cambio climático y de desnutrición (CIP, 2020).

\section{Lecciones aprendidas}

Hacer uso de las nuevas oportunidades del mercado requiere una serie de otras innovaciones, incluidas las institucionales y tecnológicas. El enfoque utilizado para mejorar las cadenas de valor de la papa 
nativa en los Andes podría ampliarse a otros cultivos andinos y a cultivos con potencial similar en otras regiones.

Es posible utilizar los recursos genéticos de las regiones de alta montaña para generar ingresos para los agricultores, revalorizando estos recursos como alimentos con valor cultural y ancestral en los mercados urbanos.

Los enfoques orientados a la innovación como el EPCP, requieren programas de investigación y desarrollo flexibles que permitan ajustes creativos para adaptarse a las circunstancias locales, respetando los principios básicos del enfoque.

\section{Cita testimonial}

"Mi esposo y yo tomamos la decisión juntos. Él también cree que es bueno invertir en papas nativas. Al reinvertir en semilla, este año pude plantar $2000 \mathrm{~kg}$. La mitad de los beneficios de la venta se destinará a ampliar la cosecha y la otra mitad a mejorar la vivienda. También queremos invertir en ganado". Nolberta Hinostroza Laureano, vicepresidenta de la comunidad Atacocha/Chicche, Junín, Perú.

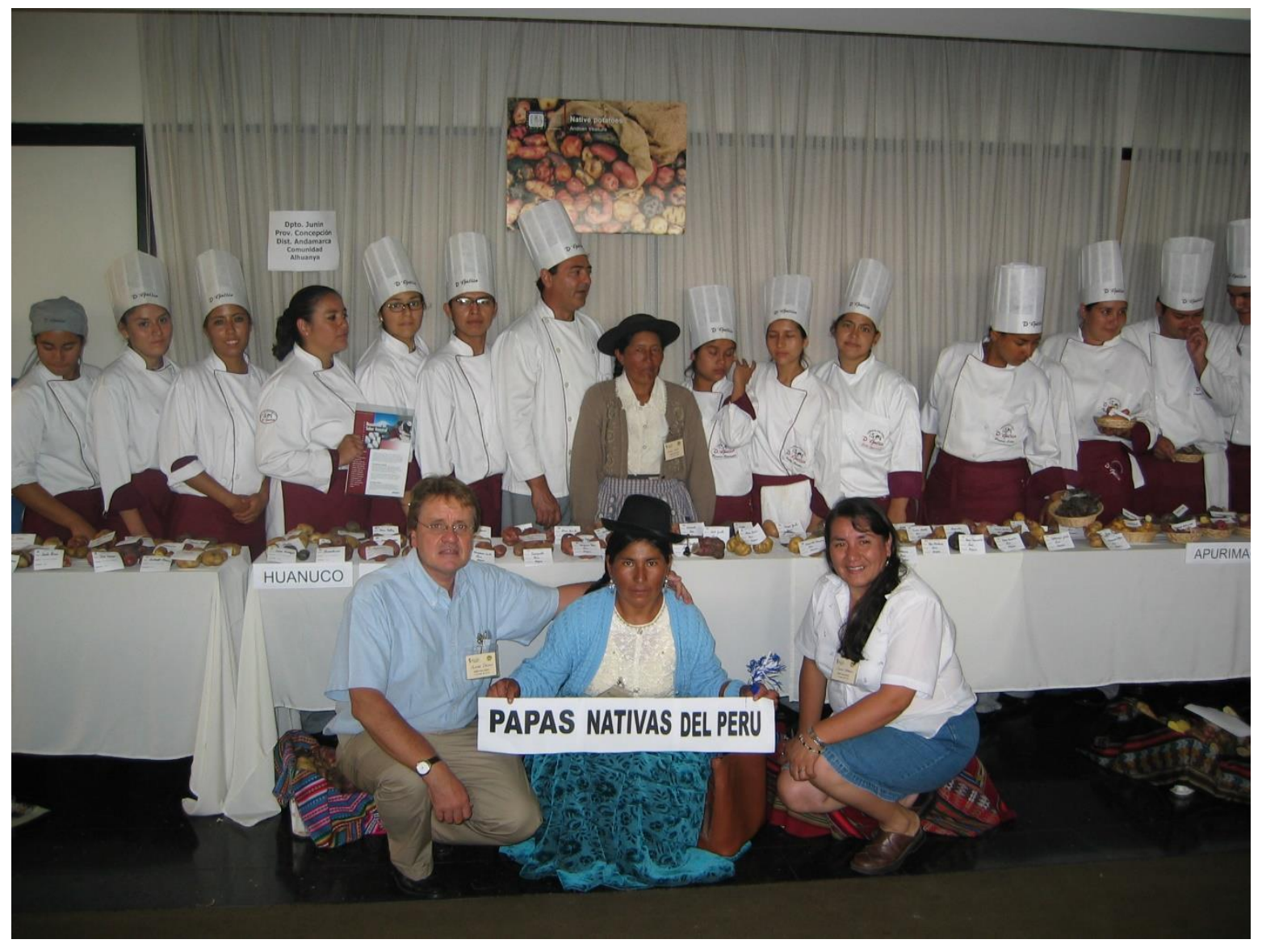

Figura 5. Colaborando con chefs para promover las papas nativas @CIP 


\section{Conflicto de intereses}

Este artículo se publicó originalmente en inglés en la Serie Sustainable Mountain Development del Centre for Development and Environment (CDE) de la Universidad de Berna en Suiza (Wymann von Dach, \& Ruiz Peyré, 2020): https://issuu.com/cde.unibe.ch/docs/vibra nt_mountain_communities_2020/1?ff.

Sin embargo, los autores declaran que el documento no presenta conflictos de intereses.

\section{Agradecimiento}

Los autores agradecen a Verónica Valcárcel por el apoyo en la traducción y edición sugerida de este artículo.

\section{Referencias citadas}

Bernet, T.; Thiele, G.; Zschocke, T.; Lopez, G.; Velasco, C.; Devaux, A. (2011). 2011. Enfoque participativo en cadenas productivas (EPCP): Manual del usuario. Lima, Perú, International Potato Center.

https://hdl.handle.net/10568/101501

Centro Internacional de la Papa (CIP). (2020). Iniciativa Andina: promoviendo una agenda de colaboración en agrobiodiversidad, acción climática y dietas saludables. Centro Internacional de la Papa (CIP), Lima, Perú. https://cgspace.cgiar.org/handle/10568/1 $\underline{08852}$

Devaux, A.; Velasco, C.; Ordinola, M.; Naziri, D. (2020). Enhancing value chain innovation through collective action: Lessons from the Andes, Africa, and Asia. In H. Campos \& O. Ortiz, eds. The potato crop, pp. 75-106. Cham, Switzerland, https://link.springer.com/ chapter/10.1007/978-3-030-28683-5_3. 55

Devaux, A.; Horton, D.; Velasco, C.; Thiele, G.; López, G.; Bernet, T.; Reinoso, I.; Ordinola, M. (2016). Collective action for market-chain innovation in the Andes. In A. Devaux; M. Torero; J. Donovan \& D. Horton, eds. Innovation for inclusive value-chain development: Successes and challenges, pp. 229-248. Washington, DC, USA, International Food Policy Research Institute. http://dx.doi. org/10.2499/9780896292130_07.

Mayanja, S.; Barone, S.; McEwan, M.; Bernet, T.; Amaya, N.; Terrillon, J.; Velasco, C.; Babini, C.; Thiele, G.; Prain, G.; Devaux, A. (2016). Prototype guide for integrating gender into Participatory Market Chain Approach. Lima, Peru, International Potato Center.

https://cgspace.cgiar.org/ handle/10568/88096.

Ordinola, M.; Devaux, A.; Manrique, K.; Fonseca, C.; Thomann, A. (2011). Strengthening competitiveness of the potato market chain: An experience in Peru. In A. Devaux, M. Ordinola \& D. Horton, eds. Innovation for development: The Papa Andina experience, pp. 151160. Lima, Peru, International Potato Center. $\quad$ http://cipotato.org/wpcontent/uploads/2013/08/005850.pdf.

Sarapura Escobar, S.; Hambly Odame, H.; Thiele, G. (2016). Gender and innovation in Peru's native potato market chains. In J. Njuki, J. Parkins \& A. Kaler, eds. Transforming gender and food security in the global South, pp. 160-185. New York, NY, USA, Routledge, International Development Research Centre. 
https://www.idrc.ca/en/book/transforming -gender-and-food-security-global-south.

Wymann von Dach, S. \& Ruiz Peyré, F., eds. 2020. Vibrant mountain communities. Regional development in mountains: Realizing potentials, tackling disparities. Bern, Switzerland, Centre for Development and Environment (CDE), University of Bern, with Bern Open Publishing (BOP). 56 pp. DOI: 10.7892/boris. 146720 